\title{
Complexité des psychothérapies et de la recherche clinique dans les services d'oncologie
}

\section{Complexity of psychotherapy and clinical research in oncology departments}

\author{
M.-F. Bacqué \\ (C) Springer-Verlag France 2010
}

\section{Les psychothérapies en oncologie ont été mises en place par les psychologues cliniciens}

Les psychologues cliniciens travaillent en cancérologie depuis les années 1970. D'abord à temps partiel puis à temps complet, ils ont construit progressivement leur pratique sur le soutien psychologique des familles d'enfants malades, l'accompagnement psychologique des enfants et des adultes malades et, enfin, les psychothérapies et les groupes de malades. Ils ont concomitamment animé des groupes de soignants (ces interventions ne seront pas traitées ici, mais elles ont aussi des effets thérapeutiques indirects sur les malades et les proches).

Les psychiatres travaillent également en oncologie depuis longtemps, mais sous une forme plus éloignée de la psychothérapie, dans un premier temps. Appelés en cas de décompensation ou de crises, pour établir des directives de traitements ou limiter des contre-indications thérapeutiques, ce n'est que récemment, depuis la création d'unités de psycho-oncologie, que les psychiatres ont pu travailler à temps plein dans les services d'oncologie et développer à leur tour leurs compétences en tant que praticiens des psychothérapies.

\section{Caractéristiques des psychologues en oncologie}

La psycho-oncologie est la première interdiscipline créée entre médecine et psychologie en France. S'il existe une spécialisation psychologique, comparativement, la neuropsychologie est une spécialité de la psychologie, mais ne recouvre pas exactement la pratique des psychologues en service de neurologie. On peut être psychologue clinicien en neurologie sans jamais faire passer de batterie de tests,

\section{M.-F. Bacqué $(\bowtie)$}

Université de Strasbourg, département de psychologie,

12, rue Goethe, F-67000 Strasbourg, France

e-mail : mfbacque@club-internet.fr tandis que l'on peut être neuropsychologue à l'hôpital général et proposer des bilans neuropsychologiques aux patients dont les fonctions cognitives sont atteintes, sans jamais « suivre » de patients en entretiens ou en psychothérapie. En revanche, sur le même modèle que celui de la psychooncologie, existe en Allemagne, la Psychokardiologie qui traite des aspects psychologiques des malades atteints d'affection cardiaque. Il y aura sans doute un jour, une psychoendocrinologie, de même qu'il pourrait y avoir une spécialité pour les psychologues travaillant en chirurgie esthétique. Le psychiste (psychologue, psychiatre ou psychanalyste) hospitalier ne peut ignorer ou mettre de côté la dimension somatique du patient, y compris en psychiatrie. Cependant, indépendamment de la maladie et de ses conséquences, son bagage théoricoclinique doit lui permettre de travailler avec tout patient, quelles que soient ses atteintes (c'est le cas des services de psychiatrie de liaison). En effet, ce sont les aspects subjectifs de la maladie et ce que le patient a à en dire qui vont être pris en compte. Cependant, tout thérapeute, dans le champ médical, sera attentif à ses propres représentations et aux spécificités de la pathologie. Ces dernières diffèrent selon qu'il travaille en oncologie, en cardiologie, en diabétologie ou en psychiatrie.

\section{Les interventions en psycho-oncologie}

Nous partons donc d'un premier constat : les personnes sont rencontrées à l'hôpital (elles supportent le poids d'une institution qui va lui-même influencer l'éventuelle demande d'entretien psychologique) ; elles présentent une maladie grave, qui remet en cause leur espérance de vie et qui est porteuse de représentations angoissantes ; enfin, elles sont soumises à des traitements lourds qui les rendent parfois ambivalentes à l'égard de la médecine et de tous les soignants.

La question de l'institution est incontournable, elle s'allège de plus en plus, cependant, en raison du raccourcissement des séjours hospitaliers. Cependant, de nombreux 
patients sont maintenant suivis à l'extérieur de l'hôpital soit par des psychistes privés, soit encore dans le cadre de prises en charge associatives ou de consultations ambulatoires à l'hôpital de référence.

La maladie cancer et ses traitements forment la deuxième caractéristique incontournable qui influence patients et soignants et modifie le cadre (temps limité par la menace de mort) et l'alliance thérapeutique (forte induction du milieu hospitalier). Cependant, dans d'autres cas, une menace de mort est sous-jacente à la psychothérapie, lorsque cette dernière est installée à la suite d'une tentative de suicide, d'une overdose ou d'une pratique anorectique extrême. Insistons sur le fait que les cancers véhiculent des images et des croyances spécifiques quant aux causes de la maladie et aux effets des thérapeutiques. Ainsi, de nombreux patients rencontrés à l'hôpital accueillent le psychologue ou psychiatre en disant qu'ils n'ont rien à perdre en acceptant l'entretien et qu'au pire, si une psychogenèse n'est pas démontrée, ils ont intérêt à mettre toutes les chances de leur côté. Les questions de l'absence de demande directe de psychothérapie, de la menace mortelle plus ou moins refoulée et du poids de la structure hospitalière jouent donc a priori contre la mise en place d'une psychothérapie en service d'oncologie.

\section{Qu'est-ce qu'une psychothérapie en oncologie ?}

Cette question est elle-même encore largement débattue, mais si nous souhaitons l'aborder en cancérologie, c'est pour tenter de trouver plus facilement un consensus que pour l'ensemble des contextes psychothérapiques, dans la mesure où deux facteurs sont communs entre nous : l'hôpital, le réseau et le cancer.

Que pourrait chercher à produire/créer une psychothérapie?

- Réduire la souffrance psychique nous semble être l'argument premier. Dans le cas des psychothérapies psychodynamiques, l'intérêt n'est pas centré sur les symptômes ou les comportements, mais porte sur le bien-être général, la compréhension de soi et les relations interpersonnelles. Ces changements qui peuvent mener, dans les psychothérapies et les psychanalyses suffisamment longues, à des modifications structurales de la personnalité s'associent indéfectiblement à la notion de temps. Il est en effet rare qu'une psychothérapie réduise immédiatement la souffrance psychique du patient, contrairement à un entretien qui peut permettre une libération "cathartique ». Paradoxalement, les premières séances sont parfois douloureuses en raison d'une prise de conscience ; cependant, dans le moyen et le long termes, les patients évoquent un sentiment de mieux-être, comme le montre la métaanalyse de Leichsering et Rabung, publiée dans JAMA en $2008[10]$;

- permettre au sujet une relative adaptation à la crise (le cancer et ses bouleversements) et à l'institution (l'hospitalisation et les prescriptions médicales). Le psychiste fait partie de l'institution, il participe aux réunions de concertation, il émet un avis qui, sans dévoiler l'intimité psychique du patient, apporte des éléments d'orientation thérapeutique à l'équipe soignante. La position en " méta » du psychiste, qui permet de se placer en recul, à distance des émotions, donne une certaine liberté au patient qui peut lui confier ses difficultés spécifiques par rapport aux hospitalisations (elles font souvent écran à d'autres difficultés inconscientes). Même si le psychiste ne donne aucune réponse d'ordre concret, le fait d'être à l'écoute de ces difficultés va permettre au patient de mieux les diriger vers les «personnes adéquates ». Ici, le psychiste n'a pas d'objectif pour ou à la place du patient. Cependant, les plaintes, les malaises confiés par les patients portent très fréquemment sur leurs difficultés à accepter, par le biais des hospitalisations, leur nouvelle vulnérabilité, leur rapprochement avec un lieu morbide (où la maladie et la mort sont présentes explicitement), leur angoisse de perte d'identité dans un univers très technique et médicalisé. Mais, la question de la relative « adaptation » comprend aussi la délicate adhésion aux soins, voire le refus de soins. Pour de rares patients, se traiter est « impossible »; cependant, ils peuvent, lors d'entretiens psychothérapiques, rechercher les raisons profondes et souvent inconscientes de cette impossibilité et les assumer plus complètement. Certains changeront alors d'avis, d'autres discuteront avec leur médecin pour améliorer les conditions de leur traitement et mieux les supporter. La psychothérapie n'a donc pas pour but d'adapter le patient aux traitements proposés par l'hôpital, mais permet au sujet d'assumer en conscience la responsabilité de ses choix de vie;

- rechercher le sens de sa vie ou le sens des bouleversements liés à la maladie. Ce n'est pas un but en soi de la psychothérapie, mais nombreux sont les patients qui posent d'emblée cet objectif dans leur psychothérapie. Ils veulent comprendre ce qui leur arrive, ils émettent des hypothèses quant à leur passé, ils sont parfois envahis par leurs questionnements anxieux et sont soulagés par la mise en mots de ces ruminations. Cette quête de sens est souvent une rationalisation qui protège de la désorganisation. Lorsque le malade éprouve l'absence de sens de sa maladie, il peut s'effondrer dans un vide dépressif que le psychiste va contenir grâce au cadre institué par la psychothérapie. Cette fonction contenante, d'abord enveloppe externe de la psyché du patient, " sécurise » sa régression. Il est souvent difficile pour les soignants 
et médecins de tolérer cet état de ralentissement alors que le mot d'ordre dû à l'emprise de l'économie sur la santé est au raccourcissement de la durée de séjour. Le psychiste ne peut maintenir un black-out sur ces phases classiques de la psychothérapie. Il doit à mon avis, expliquer un certain nombre de points techniques et éthiques de son travail afin d'intégrer les transferts latéraux des soignants qui critiquent consciemment et inconsciemment les effets immédiatement visibles de la psychothérapie (la fameuse remarque des infirmières à l'égard du psychologue qui « fait pleurer les malades »);

- redonner une certaine souplesse au fonctionnement psychique en améliorant la régulation des émotions du malade et ses réponses aux conditions de la réalité. Ce principe de la psychothérapie est commun à toutes les psychothérapies. Les psychanalystes, même s'ils ne souhaitent rien pour leurs patients (contrairement aux TCC, ils n'ont pas d'objectif ou de programme standard à proposer), reconnaissent leur action sur les processus de liaison affects-représentations, qui sont facilités par le Durcharbeitung freudien. Ce concept qui apparaît dès 1895 dans les Études sur l'hystérie et qui est traduit par Laplanche et Pontalis par " élaboration interprétative » explique le dépassement de la compréhension « intellectuelle » par le patient du matériel donné en psychothérapie pour intégrer les expériences et aboutir au changement ;

- prévenir les autres crises : une psychothérapie a des effets préventifs si le patient a réalisé ses modes de réaction passés et peut dorénavant mieux appréhender le futur. Dans le cas d'un patient atteint de cancer, cet aspect préventif est particulièrement important. Les psychothérapies pourraient faciliter l'approche de nouvelles crises comme les rechutes métastatiques, la guérison (et le fait de quitter une équipe salvatrice), la mort. Les études nous manquent ici ; cependant, de très nombreuses observations et suivis de patients nous montrent combien les psychothérapies passées ou présentes facilitent l'abord de nouveaux bouleversements liés à la maladie [12].

\section{Fondements de la formation à la pratique des psychothérapies}

Il serait difficile de passer outre l'actualité, au moment où nous écrivons ces lignes ! Le décret d'application de la $\operatorname{loi}^{1}$ (et surtout son annexe) sur les psychothérapies nous apparaît comme totalement inconséquent, pire même, véritablement nocif! Soit le législateur néglige totalement la pratique des psychologues cliniciens et des psychiatres en minimisant la formation théorique des uns et en dotant

1 Décret $\mathrm{n}^{\mathrm{O}} 2010-534$ du 20 mai 2010 relatif à l'usage des psychothérapies. les autres d'une formation ex nihilo à la psychothérapie, alors que la plupart des psychiatres passent leur internat à mener, tant que faire se peut, leur travail de médecin (prescription, suivi) et ne consacrent, sans doute à leur corps défendant, que peu de temps à se former aux psychothérapies. Des pétitions, des recours en justice, permettront de reparler de l'indignation qui précède l'application de ce décret qui, espérons-le, sera abrogé. La formation des psychologues cliniciens est pourtant d'un très bon niveau en psychopathologie. De plus, au niveau de leur pratique, dans tous les masters de psychologie clinique, ils sont en stage supervisé pendant deux ans (environ 500 heures) et font, pour les plus avancés, des psychothérapies, même si la plupart assurent plutôt des suivis (des entretiens réguliers dans un cadre spécifique). L'ensemble des psychologues cliniciens ajoutent à leur formation initiale de master, des supervisions de type analytique lorsqu'ils sont en postes, adhèrent parfois à des sociétés de psychanalyse, bénéficient d'un travail collectif dans les collèges de psychologues, ou encore dans des ateliers de formation tels que ceux de la Société française de psycho-oncologie (SFPO).

$\mathrm{Si}$ le master de psychologie clinique n'est pas copie conforme dans les différentes facultés de psychologie, on y retrouve cependant les points communs éthiques, psychanalytiques, psychopathologiques, méthodologiques et enfin de recherche clinique.

Notre remarque portera plus précisément sur deux points constamment discutés :

- non, la supervision des professionnels français n'est pas obligatoire (comme en Allemagne où elle est au minimum de trois années);

- non, ce travail sur les cas singuliers en groupe ou auprès de « seniors » n'est pas pris en charge par l'institution (sauf dans des cas particuliers, je ne peux m'empêcher de citer notre cas à l'Hôtel-Dieu de Paris, dans le service du Pr Robert Zittoun : les trois psychologues du service d'hématologie ont eu, pendant l'ensemble de leur expérience, la chance insigne (!) de bénéficier de la supervision, deux heures par semaine par un psychanalyste « senior », de pouvoir mener des recherches et de publier [1] déjà dans les congrès de la SFPO (à l'époque encore « Psychologie et cancers »).

\section{Formation continue des psychologues cliniciens et des psychiatres}

Jusqu'à présent, les psychologues cliniciens doivent payer leur supervision professionnelle et leur formation complémentaire. Cela ne devrait plus avoir lieu et les institutions devraient veiller à ce que psychologues et psychiatres puissent trouver le temps nécessaire à ces supervisions ou ces partages de cas et les payer au titre de la formation complémentaire. 
Ce training postmaster ou postdoctorat devrait être inclus dans tout emploi d'un psychologue ou d'un psychiatre susceptibles de mettre en place des psychothérapies. Certains d'entre nous « profitent» de la recherche clinique pour trouver des collègues avec qui partager leurs cas, critiquer et analyser leurs transferts, c'est ce que Michael Balint appelait le training cum research et qui constitue à notre avis, le minimum minimorum nécessaire à la pratique de psychothérapies hospitalières.

\section{La question de l'alliance thérapeutique}

Ce concept est issu de la psychanalyse. Zetzel [15] a introduit pour la première fois ce concept en 1956, alors qu'elle évoquait le transfert et la résistance en analyse. Pour elle, une partie du transfert initial est utilisée comme « alliance thérapeutique » entre l'analyste et son patient. Cette alliance est formée de l'attachement du patient au thérapeute et de l'identification à ce dernier. Greenson [6] propose dans le même registre la notion "d'alliance de travail » pour évoquer la collaboration, à parts égales entre analyste et patient : « L'alliance de travail est cette relation relativement non névrotique et rationnelle qui s'établit entre patient et analyste et qui donne au patient la possibilité de travailler efficacement dans la situation analytique ». Bordin [3] généralisera, en 1975, cette définition à tous les types de psychothérapies. Paul Gerin et Alice Dazord [4] seront les seuls à adapter un questionnaire d'alliance thérapeutique en français. Ils montreront, comme la plupart des études anglo-saxonnes, la valeur prédictive de l'alliance précoce dans les psychothérapies institutionnelles, à condition de séparer les types de pathologies des types de prise en charge (les schizophrènes sont plus améliorés par les thérapies institutionnelles, les patients avec des troubles de la personnalité dans des thérapies de groupe). Cette question de l'alliance de travail met en évidence un critère difficile à accepter : si patient et psychothérapeute doivent construire ensemble une alliance, comment ne rencontreraient-ils pas les injustices du hasard qui conduiraient tel psy à travailler dans telle institution, alors que tel patient « s'apparierait » tellement mieux avec tel autre ? C'est ici que les qualités thérapeutiques du psychiste constituent un socle commun pour tous ceux qui souhaitent les développer (leur utilisation doit être consciente et éthique, car elles peuvent donner lieu à des usurpations et des manipulations. Il s'agit aussi tout simplement du fameux effet placebo). Les qualités du thérapeute sont de plusieurs ordres :

- qualités personnelles d'intégrité psychique (absence de problèmes émotionnels) et maturité ;

- qualités interpersonnelles de compréhension, authenticité, acceptation de l'autre, implication et compréhension empathique :
- compétences professionnelles : qualités d'expertise et de justesse interprétative. L'expérience clinique du thérapeute est ici liée non pas au nombre d'années de pratique, mais au nombre et à la variété de patients traités.

Dans les différentes études portant sur les facteurs principaux de résultats dans les psychothérapies, la plupart montrent que ce n'est pas tant la nature de la technique utilisée, mais que les qualités personnelles du thérapeute et la qualité de l'alliance thérapeutique $[9,5]$ sont premières. Luborsky [11] a détaillé les deux types d'alliances thérapeutiques qui s'appliquent bien, en outre, aux psychothérapies menées à l'hôpital avec une demande quasiment inexistante au début (alliance aidante où le thérapeute est d'abord perçu comme un soutien chaleureux) et les psychothérapies en libéral, où l'alliance est perçue comme une situation de travail aux responsabilités partagées entre thérapeute et patient. Ces résultats d'études doivent être maniés avec précaution et resitués dans leur contexte (ne commettons pas les mêmes erreurs que la malencontreuse méta-analyse de l'Inserm $^{2}$ en 2004). Il est impossible de standardiser les psychothérapies et encore moins les thérapeutes et leurs patients ! Dans le précieux livre dirigé par Georges Fischman, Robert [13], psychiatre des hôpitaux et membre de la Société psychanalytique de Paris, souligne dans une boutade que « La bonne intervention thérapeutique est celle proposée par un thérapeute doué, à savoir celui qui sait intégrer le savoir et l'empathie, à un patient souhaitant la recevoir...».

Les facteurs de succès thérapeutique sont d'abord liés au patient [7]. Ainsi, le degré d'engagement du sujet s'avère déterminant dans l'amélioration thérapeutique. L'insight, c'est-à-dire la volonté de compréhension et la capacité de changement, relève aussi de cet engagement. Mais la personnalité du patient, les événements de sa vie, ses relations interpersonnelles ont des conséquences indéniables sur le déroulement de la psychothérapie. L'évolution naturelle du patient peut également être un facteur de rémission spontanée, comme le montre le fameux effet «mise sur une liste d'attente », mais attention, les patients « sur liste d'attente » ont toujours, pour des raisons éthiques, demandé à faire une psychothérapie.

\section{Appel à recherches cliniques sur les psychothérapies en oncologie}

Les développements du thème des psychothérapies sont infinis, mais une voie pragmatique pourrait être mise en place, avec beaucoup de bonne volonté, et surtout en profitant du réseau des psychistes en oncologie. Et pourquoi pas

\footnotetext{
2Rapport d'évaluation sur l'efficacité comparée des psychothérapies, Inserm, 2004
} 
lancer un appel aux psychologues, psychiatres et psychanalystes adhérents de la SFPO ?

Lors de ma formation à l'institut de psychosomatique, je participais déjà à des recherches sur les psychothérapies psychanalytiques de malades somatiques. Le dispositif consistait à demander l'autorisation à un patient, souhaitant un « traitement » psychanalytique, d'être filmé pendant sa séance. En temps réel, cette séance était retransmise en vidéo au groupe de psychanalystes en formation. Ceux-ci cotaient, de façon qualitative, une fiche à la fois diagnostique et prédictive d'items décrivant le patient et l'interaction avec son analyste. Le psychanalyste qui avait eu la séance avec son patient revenait dans le groupe immédiatement après et discutait avec lui des éléments conscients et inconscients (lorsqu'ils surgissaient) de cette heure passée avec le patient. Il annotait sa fiche à son tour. Pour chaque patient, la fiche diagnostique s'affinait au fur et à mesure des séances, dans une certaine concordance, tandis que les aspects thérapeutiques étaient observés et notés au long de la psychothérapie analytique par le groupe.

Thurin et Thurin [14] proposent, de la même façon, des autoévaluations réciproques de praticiens dans des supervisions collectives régulières de quatre ou cinq thérapeutes exposant leur clinique. À partir d'un enregistrement audio ou écrit in extenso (possible en séance d'analyse), les cliniciens remplissent (parmi d'autres questionnaires) le Psychotherapy Process Q-Set (PQS) élaboré par Jones et Windholtz en1990 [8]. Il s'agit de 100 items décrivant :

- l'attitude et le comportement du patient ;

- les actions ou les attitudes du clinicien;

- le climat et les interactions de la dyade.

Cette recherche pilote, publiée en 2007, permet à la fois d'organiser une grande étude clinique en réseau de pairs, mais de plus, elle permet a minima de former en « intervision » les praticiens de psychothérapie, dans un registre plus spécifique. Dans le cas de la psycho-oncologie, on imagine comment cette méthode génère une base de données cliniques fiable (validée avec une grande concordance) et des groupes de pairs qui affinent mutuellement leur méthode, leur éthique, leur suivi de cas singuliers.

D'autres chercheurs en psychanalyse suivent d'autres modèles [2], comme par ailleurs d'autres pratiques psychothérapiques sont également répertoriées (en particulier dans le domaine des thérapies cognitivocomportementales). Cependant, thérapies analytiques et cognitivocomportementales ne partagent pas les mêmes objectifs, en particulier lorsque le thérapeute envisage "d'éduquer » le patient ou de lui « expliquer » son fonctionnement.

La richesse des études et la créativité des praticiens montrent que la question des psychothérapies est en plein devenir. Le champ de la psycho-oncologie n'est que depuis récemment sensible à la dimension collective d'un partage des connaissances sur les psychothérapies. Ces psychothérapies sont cependant mises en place depuis plus de 30 ans. Souhaitons que ce numéro ouvre une ère nouvelle de mise en commun des connaissances et des pratiques de chacun afin que le travail effectué par les praticiens de la psyché soit encore mieux reconnu dans le domaine de l'oncologie, mais, de façon plus vaste dans celui de la souffrance psychique.

Remerciements : l'auteur remercie Sylvie Pucheu et Patrice Cannone pour leur relecture attentive et motivée.

Conflit d'intérêt : l'auteur déclare ne pas avoir de conflit d'intérêt.

\section{Références}

1. Bacqué MF, Kipman SD, Rusznievski M, et al (1984) Réflexions à partir de l'introduction de psychistes dans un service d'hématologie. Psychol Med 16(13):2247-9

2. Baruch C, Gibeault A, Loukomskaîa M (2005) Évaluation de psychothérapies psychanalytiques : une étude pilote. Cliniques Méditerrannéennes 71:259-67

3. Bordin ES (1975) The generalizability of the psychoanalytic concept of the working alliance. Psychother Theory Resp Pract 976(16):252-75

4. Dazord A (1994) Comment évaluer les psychothérapies ? J Psychol 114:21-7

5. Despland JN, De Roten Y, Depars J, et al (2001) Contribution of patient defense mechanisms and therapist interventions to the development of early therapeutic alliance in a brief psychodynamic investigation. J Psychotherapy Pract Res 10:155-64

6. Greenson RR (1967) The technique and practice of psychoanalysis. New-York. International University Press

7. Huber W (1993) Les psychothérapies : quelle thérapie pour quel patient? Nathan Université, Paris

8. Jones EE, Windholtz M (1990) The Psychoanalytic case study: towards a method for systematic inquiry. J Amer Psychoanal Assn 38:985-1015

9. Krupnick JL, Sotsky SM, Elkin I, et al (1996) The role of the therapeutic alliance in psychotherapy and pharmacotherapy outcome: findings in the National Institute of Mental Health Treatment of Depression Collaborative Research Program. J Cons Clin Psychol 64:532-9

10. Leichsering F, Rabung S (2008) Effectiveness of long term psychodynamic psychotherapy: A meta-analysis. JAMA 300:1551-65

11. Luborsky L (1994) Therapeutic alliances as predictors of psychotherapy outcomes: factors explaining the predictive success. In Hovarth AO, Greenberg LS (eds) The working alliance: Theory, research and practice. John Wiley \& Sons, New York

12. Pucheu S (2008) L'adaptation comme processus subjectif dynamique de réorganisation psychique : l'exemple de trois patients atteints de cancer du côlon, Psycho-Oncologie 2:164-78

13. Robert M (2009) État actuel des recherches appliquées à la psychanalyse et à la psychothérapie psychanalytique. In: Fischman G (sous la dir.) L'évaluation des psychothérapies et de la psychanalyse. Fondements et enjeux. Masson, Paris

14. Thurin JM, Thurin M (2007) Évaluer les psychothérapies. Méthodes et pratiques, Dunod, Paris

15. Zetzel ER (1956) Current concepts of transference. Int J Psychoanal 37:369-76 